\title{
Diagnostic Distribution of Psychiatric Disorders among Korean Young Adults
}

\author{
Ram Hwangbo', Hyejung Chang ${ }^{2}$, and Geon Ho Bahn ${ }^{3}$ \\ ${ }^{1}$ Sungmo Clinic, Seoul, Korea \\ ${ }^{2}$ School of Business, Kyung Hee University, Seoul, Korea \\ ${ }^{3}$ Department of Psychiatry, Kyung Hee University School of Medicine, Seoul, Korea
}

\begin{abstract}
Objectives: The prevalence of psychiatric disorders among young adults is different from that among younger or older age groups because of biological and environmental changes. The purpose of this study was to analyze the diagnostic distribution of psychiatric disorders in 19-30-year-old Koreans based on their age and gender using data from the Korean National Health Insurance Service (NHIS). Methods: From the 2011 medical claims sample data of NHIS of 1,375,842 people, we extracted the data of 221,038 people aged 19-30 years, including 106,232 (48.1\%) men and 114,806 (51.9\%) women. We evaluated the overall changes in the diagnostic distribution of psychiatric disorders over a 3-year period.

Results: The diagnostic frequency in women was 13,627 (59.0\%), which was significantly higher than that in men. "Other anxiety disorders" was the most common psychiatric disorder in both genders, followed by depressive episodes, somatoform disorders, "other neurotic disorders," and nonorganic sleep disorders. In men, attention-deficit/hyperactivity disorder or intellectual disabilities were not among the top 10 disorders. In women, no significant changes in major psychiatric disorders were seen over the 3-year period.

Conclusion: These results reveal the trends of diagnostic distribution of mental illnesses depending on the development, particularly in young adulthood. It is necessary to identify whether such trends are due to biological or environmental factors, aging processes, or complex influences.
\end{abstract}

Key Words: Prevalence; Psychiatric disorder; Nationwide; Young adulthood.

Received: February 28, 2020 / Accepted: March 3, 2020

Address for correspondence: Geon Ho Bahn, Department of Psychiatry, Kyung Hee University School of Medicine, 23 Kyungheedae-ro, Dongdaemoongu, Seoul 02447, Korea

Tel: +82-2-858-8556, Fax: +82-2-957-1997, E-mail: mompeian@khu.ac.kr

\section{INTRODUCTION}

Young adulthood is a period marking the end of adolescence and beginning of adulthood, during which individuals independently set the direction of their lives [1]. Although it has increasingly delayed because of industrialization, young adulthood may involve an expansion of individual problems to those affecting families and social organizations, such as marriage- and work-related issues [1]. Therefore, it is a period in which individuals are exposed to a new environment, and consequently, mental health problems may differ from those in previous stages of life. Structural maturation of the brain and hormones induce changes in the subcortical and cortical activities throughout childhood, adolescence, and adulthood. Further, as people grow out of childhood, when motor symptoms were the most prevalent, and pass through ado-

This is an Open Access article distributed under the terms of the Creative Commons Attribution Non-Commercial License (https://creativecommons.org/licenses/by-nc/4.0) which permits unrestricted non-commercial use, distribution, and reproduction in any medium, provided the original work is properly cited. lescence, psychiatric symptoms come to prevail in adulthood [2]. A study using a dual system model of brain development in adolescence, including young adulthood, suggested that this period may be marked by higher sensory seeking and lower impulse control compared to other periods [3].

Although adolescence and young adulthood are continuous phases, it is highly likely that adolescents fall into a socalled "twilight zone" [4], in which there is a great possibility of important treatments being stopped because of changes in the healthcare system, lifestyle, and environment, consequently changing the distribution of mental disorders. Nevertheless, studies investigating the distribution of mental disorders throughout one's life are rare. In surveys on the prevalence of mental disorders among adolescents aged 13-17 years using the National Comorbidity Survey (NCS) Replication Adolescent Supplement [5] and prevalence of mental disorders in the general population above the age of 18 years using the NCS Replication (NCS-R) [6], anxiety and behavioral disorders were the most prevalent mental disorders until adoles- 
cence, yet the prevalence of depressive disorders exceeded that of behavioral disorders in adulthood. Similar to these surveys conducted in the United States, a nationwide mental disorder survey has been conducted every 5 years since 2001 in Korea. This survey reported alcohol abuse (9.2\%), alcohol dependence (7.0\%), major depressive disorder (5.6\%), specific phobia (3.8\%), and generalized anxiety disorder (1.6\%) to be the most common mental disorders [7]. However, it included the general population aged 18-64 years.

Our previous study revealed interesting changes in the diagnostic distribution of mental disorders among children and adolescents under the age of 19 years based on gender and age [8]. Attention-deficit/hyperactivity disorder (ADHD) was the most prevalent mental disorder among boys aged $4-15$ years and girls aged 7-12 years. In late adolescence, anxiety disorder was the most prevalent mental disorder among both boys and girls, and girls also showed a marked increase in the prevalence of depressive disorder. In a previous study, we emphasized on the need to examine changes that occur during transition from adolescence to adulthood [8]. Therefore, the aim of this study was to analyze the diagnostic distribution of psychiatric disorders in young adulthood using the nationally representative health insurance sample data.

\section{PARTICIPANTS AND METHODS}

\section{Participants}

This study utilized the 2011 Health Insurance Review and Assessment Service-National Patient Sample (HIRA-NPS2011-0082) based on the medical claims data. The HIRA-NPS data includes $3 \%$ of the total Korean population, i.e., 1,375,842 people. The HIRA-NPS data are statistically sampled secondary data without personal identifiers, containing medical claims over a 1-year period.

We extracted the HIRA-NPS data of individuals aged 1930 years for two reasons. First, we have analyzed the diagnostic distribution of psychiatric disorders in children and adolescents aged below 19 years in a previous study [8] and have also attempted to examine changes in people aged 19 years or older in a continuum. Second, as the incidence of physical diseases begins to increase after the age of 30 years [9], distinguishing the influence of physical-mental comorbidity becomes difficult [10]. In the NPS data, 221,038 people were aged 19-30 years, including 106,232 (48.1\%) men and 114,806 (51.9\%) women. Among them, we included those who received an outpatient or inpatient treatment at a primary, secondary, or tertiary medical institution for mental disorders with an $\mathrm{F}$ (F00-F99) code per the Korean standard classification of diseases [11], which was developed based on the International Classification of Diseases (ICD) [12]. We included all codes for psychiatric disorders per person. A total of 16,297 people, including 6,417 (39.4\%) men and 9,880 (60.6\%) women, had one major diagnosis with an $\mathrm{F}$ code. When $\mathrm{F}$ codes for an individual were counted separately and the same F code was counted only once, the total number of accumulated patients were 23,102, including 9,475 (41.0\%) men and 13,627 (59.0\%) women (Fig. 1).

\section{Methods}

In the first preliminary analysis, we examined the distribution over a 1-year period. We performed another analysis over a 3-year period to examine the overall trend and found no marked differences in the results between the two analyses. Subsequently, we compared changes over a 3-year period for 23,102 people with an F-code diagnosis based on their gender. The following diagnoses had been made at a frequency of $1 \%$ or more in the preliminary analyses, and therefore, their distributions were analyzed separately from other diagnoses: mental and behavioral disorders due to alcohol use (F10), schizophrenia (F20), bipolar affective disorder (F31), depressive episode (F32), recurrent depressive disorder (F33), persistent mood disorder (F34), phobia (F40), other anxiety disorders (F41), obsessive-compulsive disorder (F42), reaction to severe stress and adjustment disorder (F43), somatoform disorders (F45), other neurotic disorders (F48), and nonorganic sleep disorders (F51). Mental retardation codes (F70, F71, F72, F73, F78, and F79) were processed as a single code (F70-79). Other mood disorders (F38) and nonspecific mood disorders (F39) were also considered similar and therefore processed as a single code. The remaining disorders were classified as "others." Therefore, 15 single diagnostic codes and one "others" code were analyzed. This study protocol was approved by the institutional review board of the Kyung Hee University Hospital (KMC IRB 1605-10).

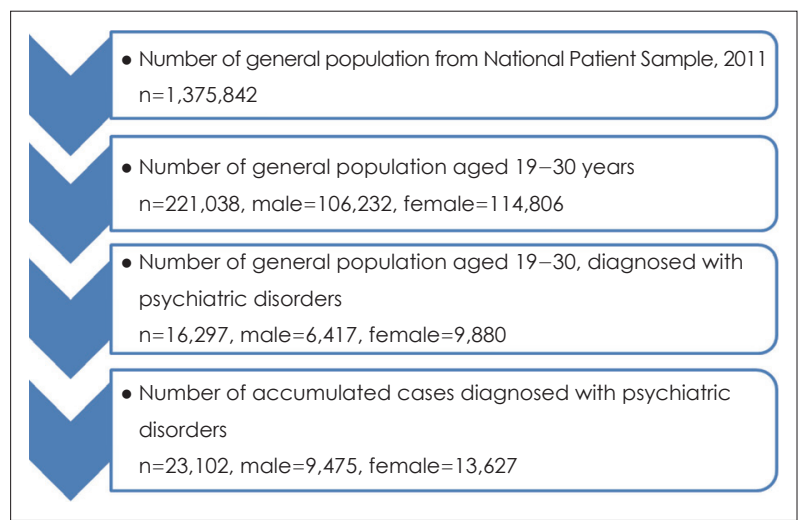

Fig. 1. Flow of the study patients and cases in analysis of distribution about psychiatric disorders. 


\section{Data analysis}

Data were analyzed with SAS 9.3 (SAS Institute Inc. Cary, NC, USA), and the distribution of mental disorders among young adults aged 19-30 years by age and gender were analyzed with the frequency analysis. A p-value less than 0.05 was deemed statistically significant.

\section{RESULTS}

\section{Number of diagnosis per subject}

The number of F-code diagnoses for each individual ranged from one to ten (Table 1).

\section{Most common diagnoses in the overall study population}

In the overall study population aged 19-30 years, the most common diagnosis was "other anxiety disorders" (F41, 24.80\%), followed by depressive episodes (F32, 14.82\%), somatoform disorders (F45, 11.31\%), "other neurotic disorders" (F48, 9.26\%), and nonorganic sleep disorders (F51, 9.19\%) (Table 2). Among the disorders included in the "others" category, the most common diagnosis was specific personality disorder ( $\mathrm{F} 60, \mathrm{n}=172)$, followed by eating disorders (F50, $\mathrm{n}=159$ ), and unspecified nonorganic psychosis (F29, $\mathrm{n}=154)$ (Table 3).

\section{Distribution by age in men}

The most common diagnosis was "other anxiety disorders" (F41), followed by depressive episodes (F32), somatoform disorders (F45), "other neurotic disorders" (F48), and nonorganic sleep disorder (F51) in both age groups of 19-21 and 22-24 years, showing the same distribution as the top six disorders (Fig. 2). The order of the most common diagnosis in the age group of 25-27 years began to change after the top three disorders, "other anxiety disorders" (F41), depressive episodes (F32), and nonorganic sleep disorder (F51), but distributions of the top five disorders in the age groups of 28-30 and 19-

Table 1. Number of psychiatric diagnoses per patient

\begin{tabular}{ccc}
\hline $\begin{array}{c}\text { Number of } \\
\text { diagnoses }\end{array}$ & $\begin{array}{c}\text { Number of } \\
\text { Patient (\%) }\end{array}$ & $\begin{array}{c}\text { Cumulative } \\
\text { frequency }(\%)\end{array}$ \\
\hline 1 & $12,185(74.77)$ & $12,185(74.77)$ \\
2 & $2,519(15.46)$ & $14,704(90.23)$ \\
3 & $931(5.71)$ & $15,635(95.94)$ \\
4 & $401(2.46)$ & $16,036(98.40)$ \\
5 & $150(0.92)$ & $16,186(99.32)$ \\
6 & $69(0.42)$ & $16,255(99.74)$ \\
7 & $25(0.15)$ & $16,280(99.90)$ \\
8 & $12(0.07)$ & $16,292(99.97)$ \\
9 & $3(0.02)$ & $16,295(99.99)$ \\
10 & $2(0.01)$ & $16,297(100.00)$ \\
\hline
\end{tabular}

24 years were the same. ADHD (F90), which was the most common diagnosis in children and adolescents, was ranked 10th in the age group of 19-21 years and further down in older age groups. Mental retardation (F70-79), which was among the top 10 disorders until 27 years of age, ranked markedly down after the age of 27 years. In the age group of $28-30$ years, the order of the top 10 disorders changed, but no new disorders other than mental and behavioral disorders due to alcohol use (F10) were added.

\section{Distribution by age in women}

In women, the age groups of 19-21, 22-24, and 25-27 years showed the same order in the top nine disorders: "other anxiety disorders" (F41), followed by depressive episodes (F32), somatoform disorders (F45), "other neurotic disorders" (F48), nonorganic sleep disorder (F51), reaction to severe stress and adjustment disorder (F43), other, unspecified mood disorders (F38-39), bipolar affective disorder (F31), and schizophrenia (F20) (Fig. 3). Mental retardation (F70-79), which was ranked 10 th in the age group of 19-21 years, was replaced by persistent mood disorder (F34) in older age groups. The age groups of 28-30 and 19-27 years showed the same order in the top

Table 2. Distribution of psychiatric diagnoses among population aged 19-30 (\%)

\begin{tabular}{|c|c|c|c|}
\hline \multirow{2}{*}{$\begin{array}{l}\text { Psychiatric } \\
\text { diagnosis }\end{array}$} & \multicolumn{3}{|c|}{ Number of case* } \\
\hline & Male & Female & Total \\
\hline F41 & $2,154(22.73)$ & $3,576(26.24)$ & $5,730(24.80)^{*}$ \\
\hline F32 & $1,348(14.23)$ & $2,075(15.23)$ & $3,423(14.82)^{*}$ \\
\hline F45 & $856(9.03)$ & $1,757(12.89)$ & $2,613(11.31)^{*}$ \\
\hline F48 & $784(8.27)$ & 1,355 (9.94) & $2,139(9.26)^{*}$ \\
\hline F51 & $787(8.31)$ & $1,337(9.81)$ & $2,124(9.19)^{*}$ \\
\hline F43 & $415(4.38)$ & $624(4.58)$ & $1,039(4.50)$ \\
\hline F20 & $409(4.32)$ & $321(2.36)$ & $730(3.16)$ \\
\hline F38-39 & $299(3.16)$ & $430(3.16)$ & $729(3.16)$ \\
\hline F31 & $290(3.06)$ & $322(2.36)$ & $612(2.65)$ \\
\hline F70-79 & $313(3.30)$ & $174(1.28)$ & $487(2.11)$ \\
\hline F34 & $145(1.53)$ & $216(1.59)$ & $361(1.56)$ \\
\hline $\mathrm{F} 40$ & $182(1.92)$ & $157(1.15)$ & $339(1.47)$ \\
\hline $\mathrm{F} 42$ & $185(1.95)$ & $132(0.97)$ & $317(1.37)$ \\
\hline F10 & $162(1.71)$ & $142(1.04)$ & $304(1.32)$ \\
\hline F33 & $92(0.97)$ & $176(1.29)$ & $268(1.16)$ \\
\hline Others & $1,054(11.12)$ & $833(6.11)$ & $1,887(8.17)$ \\
\hline Total & $9,475(100.0)$ & $13,627(100.0)$ & $23,102(100.0)$ \\
\hline \multicolumn{4}{|c|}{$\begin{array}{l}\text { *p<0.05. F10: mental and behavioural disorder due to use of } \\
\text { alcohol, F20: schizophrenia, F31: bipolar affective disorder, } \\
\text { F32: depressive episode, F33: recurrent depressive disorder, } \\
\text { F34: persistent mood disorder, F38-39: other (F38), unspecified } \\
\text { (F39) mood disorder, F40: phobic anxiety disorder, F41: other } \\
\text { anxiety disorder, F42: obsessive-compulsive disorder, F43: re- } \\
\text { action to severe stress and adjustment disorder, F45: somato- } \\
\text { form disorder, F48: other neurotic disorder, F51: nonorganic } \\
\text { sleep disorder, F70-79: mental retardation }\end{array}$} \\
\hline
\end{tabular}


Table 3. Distribution of diagnoses included in 'others'

\begin{tabular}{|c|c|c|c|}
\hline \multirow{2}{*}{ Psychiatric diagnosis } & \multicolumn{3}{|c|}{ Number of case* } \\
\hline & Male & Female & Total \\
\hline FOO & 1 & 0 & 1 \\
\hline F01 & 13 & 9 & 22 \\
\hline F02 & 1 & 0 & 1 \\
\hline F03 & 5 & 1 & 6 \\
\hline F05 & 8 & 4 & 12 \\
\hline F06 & 67 & 64 & 131 \\
\hline F07 & 44 & 33 & 77 \\
\hline F09 & 8 & 1 & 9 \\
\hline $\mathrm{F} 11$ & 2 & 1 & 3 \\
\hline F13 & 3 & 12 & 15 \\
\hline $\mathrm{F} 17$ & 7 & 8 & 15 \\
\hline F18 & 3 & 0 & 3 \\
\hline F19 & 7 & 19 & 26 \\
\hline F21 & 19 & 16 & 35 \\
\hline F22 & 18 & 17 & 35 \\
\hline F23 & 33 & 26 & 59 \\
\hline F25 & 43 & 55 & 98 \\
\hline F28 & 34 & 19 & 53 \\
\hline F29 & 100 & 54 & $154^{*}$ \\
\hline F30 & 17 & 17 & 34 \\
\hline F44 & 16 & 6 & 22 \\
\hline F50 & 20 & 139 & $159^{*}$ \\
\hline F52 & 20 & 3 & 23 \\
\hline F53 & 0 & 8 & 8 \\
\hline F54 & 40 & 98 & 138 \\
\hline F55 & 0 & 3 & 3 \\
\hline F59 & 0 & 16 & 16 \\
\hline F60 & 104 & 68 & $172^{*}$ \\
\hline F61 & 2 & 1 & 3 \\
\hline F62 & 1 & 2 & 3 \\
\hline F63 & 42 & 11 & 53 \\
\hline F64 & 12 & 2 & 14 \\
\hline F65 & 1 & 0 & 1 \\
\hline F66 & 1 & 0 & 1 \\
\hline F68 & 2 & 2 & 4 \\
\hline F69 & 3 & 2 & 5 \\
\hline F80 & 9 & 2 & 11 \\
\hline F81 & 4 & 2 & 6 \\
\hline F83 & 1 & 2 & 3 \\
\hline F84 & 102 & 18 & 120 \\
\hline F88 & 1 & 0 & 1 \\
\hline F89 & 2 & 0 & 2 \\
\hline F90 & 100 & 42 & 142 \\
\hline F91 & 17 & 5 & 22 \\
\hline F92 & 10 & 10 & 20 \\
\hline F93 & 8 & 7 & 15 \\
\hline F95 & 62 & 10 & 72 \\
\hline
\end{tabular}

Table 3. Distribution of diagnoses included in 'others' (continued)

\begin{tabular}{cccc}
\hline \multirow{2}{*}{ Psychiatric diagnosis } & \multicolumn{3}{c}{ Number of case* } \\
\cline { 2 - 4 } & Male & Female & Total \\
\hline F98 & 14 & 9 & 23 \\
F99 & 27 & 9 & 36 \\
Total & 1,054 & 833 & 1,887 \\
\hline
\end{tabular}

${ }^{*} \mathrm{p}<0.05$. F00: dementia in Alzheimer's disease, F01: vascular dementia, F02: dementia in other diseases classified elsewhere, F03: unspecified dementia, F05: delirium, not induced by alcohol and other psychoactive substances, F06: other mental disorders due to brain damage and dysfunction and to physical disease, F07: personality and behavioural disorders due to brain disease, damage and dysfunction, F09: unspecified organic or symptomatic mental disorder, F11: mental and behavioural disorders due to use of opioids, F13: mental and behavioural disorders due to use of sedatives or hypnotics, F17: mental and behavioural disorders due to use of tobacco, F18: mental and behavioural disorders due to use of volatile solvents, F19: mental and behavioural disorders due to multiple drug use and use of other psychoactive substances, F21: schizotypal disorder, F22: persistent delusional disorders F23: acute and transient psychotic disorders, F25: schizoaffective disorders, F28: other nonorganic psychotic disorders, F29: unspecified nonorganic psychosis, F30: manic episode, F44: dissociative disorders, F50: eating disorders, F52: sexual dysfunction, not caused by organic disorder or disease, F53: mental and behavioural disorders associated with the puerperium, NEC, F54: psychological and behavioural factors associated with disorders or diseases classified elsewhere, F55: abuse of non-dependence-producing substances, F59: unspecified behavioural syndromes associated with physiological disturbances and physical factors, F60: specific personality disorders, F61: mixed and other personality disorders, F62: enduring personality changes, not attributable to brain damage and disease, F63: habit and impulse disorders, F64: gender identity disorders, F65: disorders of sexual preference, F66: psychological and behavioural disorders associated with sexual development and orientation, F68: other disorders of adult personality and behavior, F69: unspecified disorder of adult personality and behavior, F80: specific developmental disorders of speech and language, F81: specific developmental disorders of scholastic skills, F83: mixed specific developmental disorders, F84: pervasive developmental disorders, F88: other disorders of psychological development, F89: unspecified disorder of psychological development, F90: hyperkinetic disorders, F91: conduct disorders, F92: mixed disorders of conduct and emotions, F93: emotional disorders with onset specific to childhood, F95: tic disorders, F98: other behavioural and emotional disorders with onset usually occurring in childhood and adolescence, F99: unspecified mental disorder

three disorders but different orders in the remaining disorders of the top 10 list, without the addition of any new diagnosis. For example, schizophrenia (F20) ranked higher while other, unspecified mood disorders (F38-39) ranked lower.

\section{DISCUSSION}

In this analysis, the most notable characteristic of mental disorders in young adulthood was the reversal of the maleto-female ratio among detected patients: more diagnoses in men than in women during childhood and adolescence and more diagnoses in women than in men during young adult- 


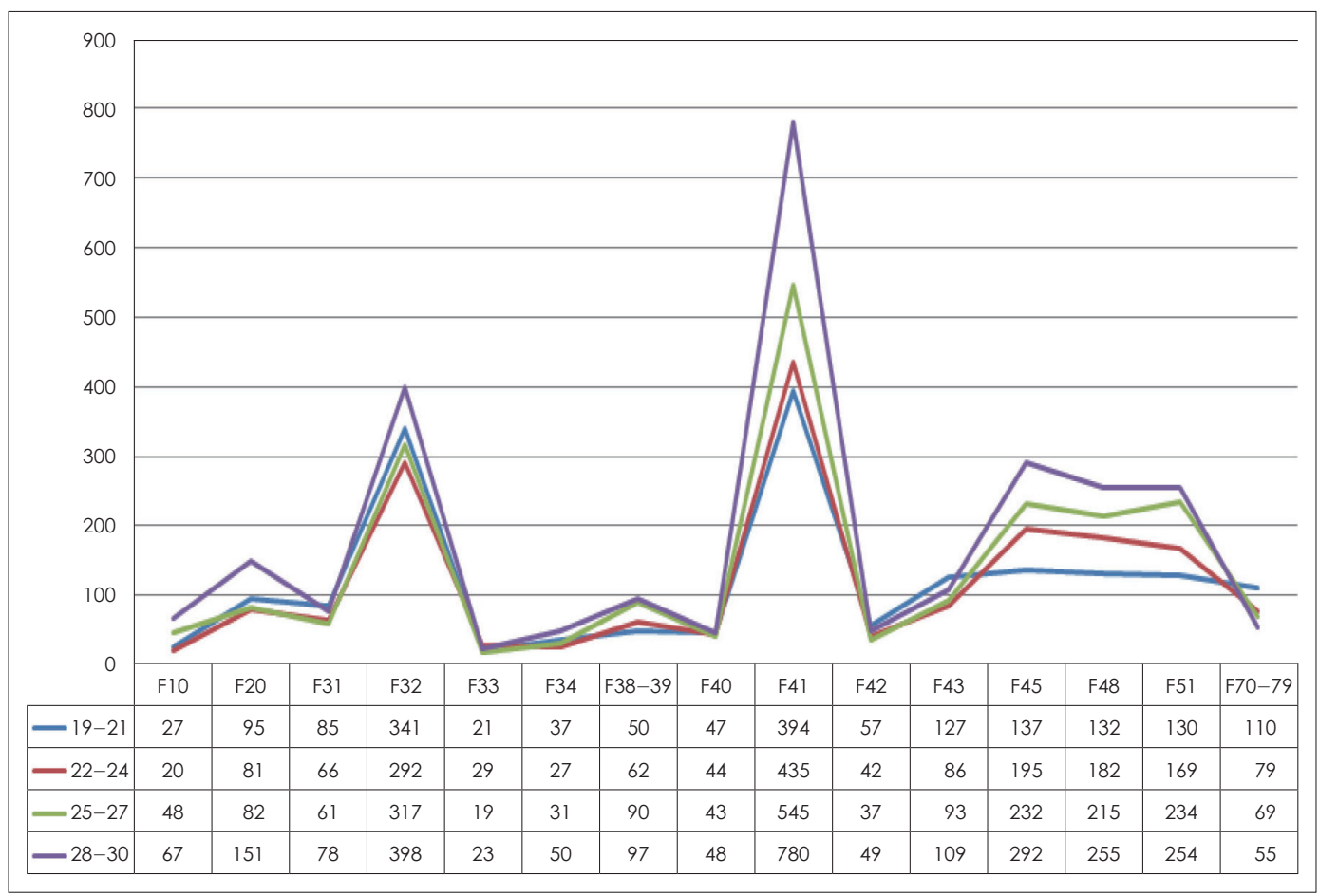

Fig. 2. Diagnostic distribution according to age (male). F10: mental and behavioural disorder due to use of alcohol, F20: schizophrenia, F31: bipolar affective disorder, F32: depressive episode, F33: recurrent depressive disorder, F34: persistent mood disorder, F3839: other (F38), unspecified (F39) mood disorder, F40: phobic anxiety disorder, F41: other anxiety disorder, F42: obsessive-compulsive disorder, F43: reaction to severe stress and adjustment disorder, F45: somatoform disorder, F48: other neurotic disorder, F51: nonorganic sleep disorder, F70-79: mental retardation.

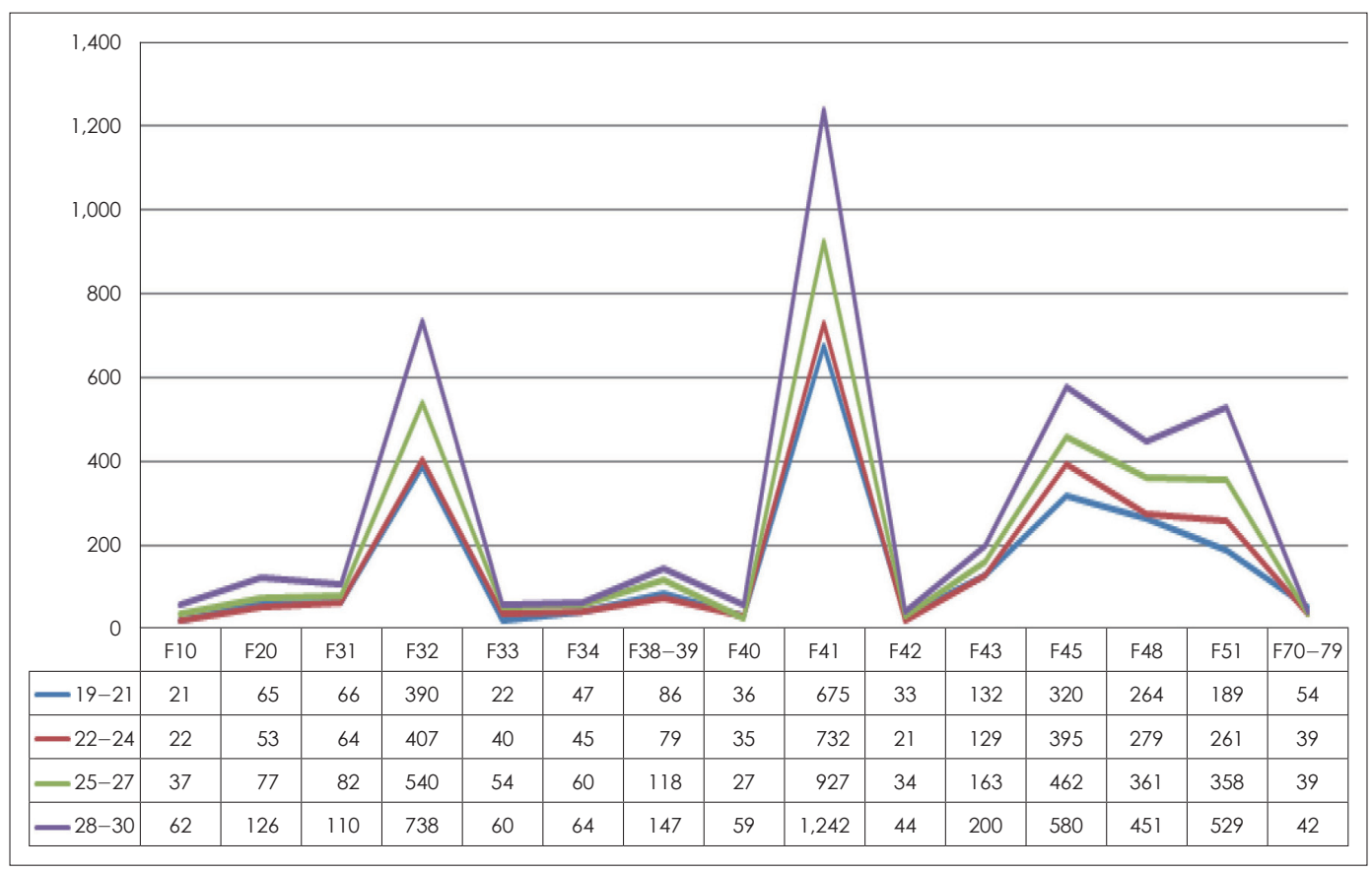

Fig. 3. Diagnostic distribution according to age (female). F10: mental and behavioural disorder due to use of alcohol, F20: schizophrenia, F31: bipolar affective disorder, F32: depressive episode, F33: recurrent depressive disorder, F34: persistent mood disorder, F38-39: other (F38), unspecified (F39) mood disorder, F40: phobic anxiety disorder, F41: other anxiety disorder, F42: obsessive-compulsive disorder, F43: reaction to severe stress and adjustment disorder, F45: somatoform disorder, F48: other neurotic disorder, F51: nonorganic sleep disorder, F70-79: mental retardation. 
hood. A cross-national meta-analysis of mental disorders also revealed that the prevalence of mental disorders is 1.1 times higher in women than in men [13].

The prevalence of mental disorders in young adulthood differs with the gender, age group, and type of disorder. In a meta-analysis, the prevalence of major depressive disorder was high among women while that of externalizing disorder was high among men [13]. While the prevalence of mental disorders was markedly higher in men than in women before the age of 19 years in our previous analysis of psychiatric disorders during childhood and adolescence, primarily due to the high ADHD prevalence among boys [8], the present study revealed that the number of women with depressive and anxiety disorders increased substantially and exceeded the number of men. The number of patients with depressive and anxiety disorders also increased with increasing age from 19 to 30 years. The increase in number of adults with anxiety and depression begins around the age of 13 years and continues into adulthood [8]. This strongly suggests that the high prevalence of anxiety and mood disorders in young adulthood in our study reflects the diagnostic continuity of mental disorders from adolescence to young adulthood. This is an issue that must be adequately addressed in studies on the prevalence of psychiatric disorders in adulthood and relevant policymaking.

A 2011 epidemiological study in Korea that utilized the Diagnostic and Statistical Manual of Mental Disorders (DSM; 4th Edition) [14] found a high prevalence of anxiety and mood disorders among women, similar to our results, but nicotine and alcohol use disorders were highly prevalent among men and younger age groups [15]. Similarly, the NCS-R that utilized DSM-IV also revealed that the annual prevalence of anxiety and mood disorders was the highest while that of substance use disorder was the second highest in the young adult population aged 18-29 years [16]. In our study, among men, both the number and percentage of cases of bipolar affective disorder (F31), depressive episodes (F32), compulsive disorder (F42), and response to severe stress and adjustment disorder (F43) dropped in the interval between 19-21 and 2224 years but began to rise again towards old age. We speculate that this is a transient decline caused by failure to present to a hospital because of mandatory military duty among Korean men in this period, as opposed to a decline in the actual occurrence of these disorders. The difference may also be attributable to the fact that the two aforementioned studies examined the general population while we used measurements from people who presented at a hospital. In other words, resulting from a cultural lenience towards smoking and drinking, people do not recognize them as an illnesses and therefore do not present to a hospital, which increases the possibility of underestimation in the medical claims data of NHIS. The fact that both the United States NCS-R [6] and Korean epidemiological study [7] used DSM-IV [14] as reference while we used ICD-10 [12] may also have contributed to such differences.

In our previous study [8], $68.8 \%$ of children and adolescents had been diagnosed with a single disorder, but in the present study, $74.8 \%$ of adults received a single diagnosis. This suggests that there were more patients with multiple diagnoses in childhood and adolescents than in adulthood. In consideration of previous reports that $66-76.2 \%$ of patients with ADHD have one or more comorbidities $[17,18]$, it is possible that a second diagnosis of a comorbidity accompanying ADHD, which was the most prevalent mental disorder in childhood and adolescence, might have been added. The prevalence of ADHD (F90) was notably low. In this study, the reason that the occurrence of ADHD rapidly declined in adulthood may be partly because ADHD medications are not covered by Korea's national health insurance system for people aged 19 years or older, which might have contributed to diminished treatment adherence because of the burden of treatment costs [19]. However, in 2016, this problem was resolved by expanding the coverage for adults, and as the combined prescription of ADHD medications has been permitted in December 2019, the number of ADHD diagnoses may increase in the coming years [19].

In this study, diagnostic frequencies of somatoform disorders, "other neurotic disorders," and nonorganic sleep disorder were high. Somatoform disorders and nonorganic sleep disorder are two independent disorders with different diagnostic codes, but somatoform symptoms and insomnia might be common major complaints in other mental disorders. Considering that Korean patients with depression were highly concerned with digestive symptoms and health [20], we cannot eliminate the possibility that depressive disorder may have been diagnosed as a somatoform disorder. Although insomnia was not included as a major mental disorder in a 2006 epidemiological study on sleep disorders, the prevalence of insomnia, when a broad definition was used, was high, with $22.7 \%$ in the age group of $18-24$ years and $20.8 \%$ in the age group of 25-34 years [21]. As sleep disorders are commonly accompanied by other illnesses [22], they might have been entered as a sub-diagnosis in addition to other diagnoses as opposed to being entered as a single disorder. Neurotic disorders, encompassing depression, anxiety, fear, hysteria, hypochondria, and somatoform disorders [23], are not included in the current DSM system [14] but remain in ICD-10 [12]. A study that extracted $1 \%$ of the Taiwanese population diagnosed based on ICD-9 and analyzed the health insurance data revealed a high prevalence of neurotic disorders, including "other neurotic disorders" (2.44\%), similar to our study [24]. 
However, results should be interpreted with caution, as ICD-9 and ICD-10 adopt a different concept of neurotic disorders.

This study had a few limitations. First, we only analyzed data spanning 1 year, so we could not compare the changes in the diagnostic distribution by year. Therefore, it would be necessary to utilize long-term accumulated data to examine whether mental disorders are replaced by "others" in the overall population or not and whether mental disorders change in the same person with age or not. Second, although our data were sampled from the entire population, groups of people for whom medical claims were not made were not included in the data, and diagnoses of mental disorders were analyzed only for $3 \%$ of the population, which limited the generalizability of the study results for the entire general population. Third, we could not examine the socioeconomic factors and other confounding factors that may impact treatment because of the personal information protection policy governing the NHIS data.

Nevertheless, using nationwide health insurance sample data to overcome the limitations of previous studies, using data that contains actual diagnoses made by psychiatrists and other doctors in clinical practice, and comprehensively investigating all mental disorders are strengths of this study. This study makes a significant contribution by revealing the trends of major psychiatric disorders in the transitional stage from adolescence to young adulthood.

\section{Acknowledgments}

The tables and figures presented in this article are part of Ram Hwangbo's master's thesis submitted to the Kyung Hee University School of Medicine in 2018.

\section{Conflicts of Interest}

The authors have no potential conflicts of interest to disclose.

\section{Author Contributions}

Conceptualization: Geon Ho Bahn, Ram Hwangbo. Data curation: Hyejung Chang. Investigation: Geon Ho Bahn. Methodology: Geon Ho Bahn, Hyejung Chang. Writing-statistics: Hyejung Chang. Writingoriginal draft: Ram Hwangbo. Writing-review \& editing: Geon Ho Bahn.

\section{ORCID iDs}

Ram Hwangbo https://orcid.org/0000-0003-2613-0404

Hyejung Chang https://orcid.org/0000-0002-5666-1305

Geon Ho Bahn https://orcid.org/0000-0002-3550-0422

\section{REFERENCES}

1) Bahn GH. What and how do psychotherapist consider the developmental stages in adult psychotherapy? Psychoanal 2019;30:7988.

2) Andersen SL. Trajectories of brain development: point of vulnerability or window of opportunity? Neurosci Biobehav Rev 2003;27: 3-18.

3) Shulman EP, Harden KP, Chein JM, Steinberg L. Sex differences in the developmental trajectories of impulse control and sensationseeking from early adolescence to early adulthood. J Youth Adolesc 2015;44:1-17.

4) Young S, Murphy CM, Coghill D. Avoiding the 'twilight zone': recommendations for the transition of services from adolescence to adulthood for young people with ADHD. BMC Psychiatry 2011; 11:174.

5) Kessler RC, Avenevoli S, Costello EJ, Georgiades K, Green JG, Gruber MJ, et al. Prevalence, persistence, and sociodemographic correlates of DSM-IV disorders in the National Comorbidity Survey Replication Adolescent Supplement. Arch Gen Psychiatry 2012; 69:372-380.

6) Kessler RC, Demler O, Frank RG, Olfson M, Pincus HA, Walters EE, et al. Prevalence and treatment of mental disorders, 1990 to 2003. N Engl J Med 2005;352:2515-2523.

7) Cho MJ, Chang SM, Hahm BJ, Chung IW, Bae A, Lee YM, et al. Lifetime risk and age of onset distributions of psychiatric disorders: analysis of national sample survey in South Korea. Soc Psychiatry Psychiatr Epidemiol 2012;47:671-681.

8) Hwangbo R, Chang H, Hong M, Cho S, Bahn GH. The diagnostic distribution of psychiatric disorders among the population under 19 years old: based on the National Insurance Data. J Korean Acad Child Adolesc Psychiatry 2016;27:139-145.

9) Lee GJ, Kim SK, Kim JH, Cho KJ. Prevalence rates of major chronic adult diseases among Korean employees. Korean J Clin Lab Sci 2009,41:196-207.

10) Kim JH, Chang SM, Bae JN, Cho SJ, Lee JY, Kim BS, et al. Mentalphysical comorbidity in Korean adults: results from a nationwide general population survey in Korea. Psychiatry Investig 2016;13: 496-503.

11) Korea Informative Classification of Diseases. Korean statistical classification of diseases and related health problems (KCD), 7th edition. Seoul: Korea Informative Classification of Diseases;2016.

12) World Health Organization. The ICD-10 classification of mental and be-havioural disorders: clinical descriptions and diagnostic guide-lines. Geneva: World Health Organization;1992.

13) Seedat S, Scott KM, Angermeyer MC, Berglund P, Bromet EJ, Brugha TS, et al. Cross-national associations between gender and mental disorders in the World Health Organization World Mental Health Surveys. Arch Gen Psychiatry 2009;66:785-795.

14) American Psychiatric Association. Diagnostic and statistical manual of mental disorders, 4th ed. (DSM-IV). Washington DC: American Psychiatric Association;1994.

15) Cho MJ, Seong SJ, Park JE, Chung IW, Lee YM, Bae A, et al. Prevalence and correlates of DSM-IV mental disorders in South Korean adults: the Korean epidemiologic catchment area study 2011. Psychiatry Investig 2015;12:164-170.

16) Kessler RC, Chiu WT, Demler O, Merikangas KR, Walters EE. Prevalence, severity, and comorbidity of 12-month DSM-IV disorders in the National Comorbidity Survey Replication. Arch Gen Psychiatry 2005;62:617-627.

17) Byun H, Yang J, Lee M, Jang W, Yang JW, Kim JH, et al. Psychiatric comorbidity in Korean children and adolescents with attention-deficit hyperactivity disorder: psychopathology according to subtype. Yonsei Med J 2006;47:113-121.

18) Reale L, Bartoli B, Cartabia M, Zanetti M, Costantino MA, Canevini MP, et al. Comorbidity prevalence and treatment outcome in children and adolescents with ADHD. Eur Child Adolesc Psychiatry 2017;26:1443-1457.

19) Bahn GH, Lee YS, Yoo HK, Kim E, Park S, Han DH, et al. Development of the Korean practice parameter for adult attention-deficit/hyperactivity disorder. J Korean Acad Child Adolesc Psychiatry 2020;31:5-25.

20) Roh S, Park YC. Characteristics of depression in Korea and nonpharmacological treatment. Korean J Biol Psychiatry 2006;13: 
226-233.

21) Kim BS, Jeon HJ, Hong JP, Bae JN, Lee JY, Chang SM, et al. DSMIV psychiatric comorbidity according to symptoms of insomnia: a nationwide sample of Korean adults. Soc Psychiatry Psychiatr Epidemiol 2012;47:2019-2033.

22) Roth T, Jaeger S, Jin R, Kalsekar A, Stang PE, Kessler RC. Sleep problems, comorbid mental disorders, and role functioning in the national comorbidity survey replication. Biol Psychiatry 2006;60:
1364-1371.

23) Bebbington P, Brugha T, Meltzer H, Jenkins R, Ceresa C, Farrell M, et al. Neurotic disorders and the receipt of psychiatric treatment. Int Rev Psychiatry 2003;15:108-114.

24) Chien IC, Chou YJ, Lin CH, Bih SH, Chou P. Prevalence of psychiatric disorders among National Health Insurance enrollees in Taiwan. Psychiatr Serv 2004;55:691-697. 